\title{
The Role of Non-Epistemic Values in Engineering Models
}

\author{
Sven Diekmann · Martin Peterson
}

Received: 20 May 2011/Accepted: 20 July 2011/Published online: 7 August 2011

(C) The Author(s) 2011. This article is published with open access at Springerlink.com

\begin{abstract}
We argue that non-epistemic values, including moral ones, play an important role in the construction and choice of models in science and engineering. Our main claim is that non-epistemic values are not only "secondary values" that become important just in case epistemic values leave some issues open. Our point is, on the contrary, that non-epistemic values are as important as epistemic ones when engineers seek to develop the best model of a process or problem. The upshot is that models are neither value-free, nor depend exclusively on epistemic values or use non-epistemic values as tie-breakers.
\end{abstract}

Keywords Epistemic values · Non-epistemic values · Engineering models · Value-judgments · Application of science

\section{Introduction}

In this article we argue that non-epistemic values, including moral ones, play an important role in the construction and choice of models in science and engineering. Our claim is not just a descriptive point about how scientists and engineers actually reason as they develop and assess models. We argue that our claim extends also to how scientists and engineers ought to reason. It goes without saying that this is a controversial claim. Engineers and scientists typically argue that models should be

\footnotetext{
S. Diekmann $(\bowtie)$

Fac. of Innovation Sciences, IPO 1.14, Department of Philosophy and Ethics, Eindhoven University of Technology, P.O. Box 513, 5600 MB Eindhoven, The Netherlands e-mail: s.diekmann@tue.nl

\section{Peterson}

Fac. of Innovation Sciences, IPO 1.04, Department of Philosophy and Ethics, Eindhoven University of Technology, P.O. Box 513, 5600 MB Eindhoven, The Netherlands e-mail: m.b.peterson@tue.nl
} 
entirely value-free or may at most rely on epistemic values such as simplicity, elegance and fruitfulness. ${ }^{1}$

The role of values in science has been thoroughly debated in the scholarly literature since the 1950's. Rudner (1953) famously pointed out that inductive claims about the justification of scientific hypotheses rely on epistemic valuejudgments, and a similar point was made somewhat later by Kuhn (1962, 1977). Kuhn argued that epistemic values such as simplicity, scope, theoretical elegance and fruitfulness influence choices between rival theories and models. Today it has become widely accepted among philosophers of science that value-judgements cannot be entirely eliminated from scientific reasoning.

McMullin (1982) deepened the discussion on values in science by explicitly addressing the distinction between epistemic and non-epistemic values. According to McMullin, '[a]n epistemic value is one we have reason to believe will, if pursued, help toward the attainment of... knowledge' (1982, p. 18) All other values are nonepistemic ones. There are of course many ways in which non-epistemic values may influence science as a whole. For instance, non-epistemic values influence the choice of research projects, the way scientific knowledge is used in society (Dorato 2004), and the pragmatic limitations of research methods (Steel 2010, p. 26). Nevertheless, according to a widely accepted view in contemporary philosophy of science, there is little room for non-epistemic values in scientific reasoning, i.e., in the basic construction and application of scientific theories and models. To put things briefly, the standard view is that non-epistemic values are allowed to influence scientific reasoning, 'only if epistemic values do not completely determine all aspects of scientific inference' (Steel 2010, p. 26; see also Dorato 2004, p. 59, for a similar claim).

We take it to be fairly uncontroversial that the philosophical discussion of epistemic values in science could, and should, be extended to engineering. The engineer faced with a choice between two or more alternative models also has to take values into account. ${ }^{2}$ The aim of this article is not to defend any particular view about how the engineering disciplines are, or should be, related to other disciplines; nor do we seek to articulate any precise view about how to draw the line between engineering and science. Our focus is on modelling, and especially on modelling in the engineering sciences. In what follows we shall provide examples which show that non-epistemic values play, and should play, a role in the development and application of engineering models. Our main claim is that non-epistemic values are not only "secondary values" that become important just in case epistemic values leave some issues open. Our point is, on the contrary, that non-epistemic values are as important as epistemic ones when engineers seek to develop the best model of a process or problem. The upshot of all this is that models are neither value-free, nor

\footnotetext{
1 See e.g., Walker (2009).

2 Epistemic values relevant to engineering need not be exactly the same as the epistemic values in traditional scientific disciplines. While some epistemic values, such as elegance and simplicity, are likely to be relevant in both engineering and science, a value such as scope seems to be of much less importance in engineering compared to physics or chemistry. Since engineers primarily seek to build artefacts that work in a particular context, it is less important whether the solution the engineer comes up with also works for solving similar problems in other contexts.
} 
depend exclusively on epistemic values or use non-epistemic values only as tie-breakers. By bringing attention to the role of non-epistemic values we hope to show that engineers have a non-trivial and morally relevant influence on the construction and application of models.

\section{What is an Epistemic Value?}

Let us start by taking a closer look at the distinction between epistemic and non-epistemic values. Although mentioned by Rudner and Kuhn, the more extensive analysis offered by McMullin (1982) has been particularly influential in recent years. McMullin claims that a value counts as an epistemic one, if and only if it helps to 'promote the truth-like character of science', whereas all other values are non-epistemic (1982, p. 18). However, just after he makes this claim, he adds that, '[a]n epistemic value is one we have reason to believe will, if pursued, help toward the attainment of... knowledge.'(1982, p. 18) A problem with making both these claims is that the pursuit of truth can conflict with the attainment of knowledge-promoting the truth will not always lead to the attainment of knowledge.

The conflict between the two definitions suggested by McMullin persists even if we think that truth is a necessary condition for knowledge. Consider, for instance, a value such as simplicity. McMullin explicitly claims that this is an epistemic value (1982, p. 16). We feel inclined to agree, but this triggers an inconsistency if we read his definitions literally. In many situations, epistemic agents emphasising simplicity will attain more knowledge than agents who pay no or little attention to simplicity. This is because much knowledge gained through simplicity is acquired by giving up some true but very complex beliefs. For instance, instead of trying to adopt a million true but slightly different beliefs about equally many mechanisms that shape our economic system, it might be more effective to single out a few hundred of the most central ones and turn them into knowledge by increasing their warrant. Therefore, although simplicity is a paradigmatic example of an epistemic value, it does not fit well with the first definition suggested by McMullin (promoting the truth), but quite well with the second (attainment of knowledge). We take the conclusion of this to be that there is a genuine conflict between focusing on truth and focusing on knowledge.

This point about the ambiguity in McMullin's distinction between epistemic and non-epistemic values has direct relevance for the engineering sciences. It seems very likely that engineers often have true beliefs about how a complex technical systems works. However, in some of those cases their true beliefs do not qualify as knowledge, simply because they are not sufficiently warranted. For instance, an engineer may truly believe that if he changes some parameter in his complex technical system just a little bit, then that will trigger a big change elsewhere in the system-but as long as he has no clue about why this is the case or whether there is some reliable mechanism which guarantees that this will always happen, his true belief does not qualify as knowledge because of its lack of warrant. So what is it that matters then for the definition of epistemic values: truth or knowledge? 
Some scholars probably see the acquisition of true beliefs as the most important goal of science, but the standard view among philosophers from Plato onwards is that knowledge is what ultimately matters. (The locus classicus is 'the road to Larissa' in Plato's The Republic). That said, the acquisition of propositional knowledge is not the sole epistemic goal of science. Consider, for instance, a Venn diagram. By drawing a Venn diagram we do not gain any new knowledge, but making such a diagram can still be an important epistemic goal simply because it makes it easier to grasp a complex body of knowledge. We take this to show that the epistemic goals of science can differ from context to context. Hence, a tentative definition of an epistemic value could be to widen McMullin's second suggestion a bit:

Definition 1 An epistemic value is one for which one has reason to believe that it will, if pursued, help toward the attainment of epistemic goals; all other values are non-epistemic values.

Unfortunately, it is easy to see that Definition 1, if read literally, fails to give us exactly what we want. The problem is that it now turns out to be an empirical question whether a value, such as simplicity, is an epistemic or a non-epistemic value. In some situations one may have reason to believe that simplicity will lead us to the attainment of epistemic goals, but this is not always the case. Take knowledge for example. Although many pieces of scientific knowledge are surprisingly simple, it is certainly incorrect to think that all are. Sometimes simplicity does not produce any knowledge at all. McMullin recognises this problem in passing, but ultimately accepts this context sensitive analysis of epistemic values. He writes:

When no sufficient case can be made for saying that the imposition for a particular value on the process of theory choice is likely to improve the epistemic status of the theory, ..., this value is held to be non-epistemic in the context in question. (McMullin 1982, p. 19)

In our view, McMullin's context-dependent approach to epistemic values is problematic. Arguably, which values count as epistemic should not depend on contingent facts that vary from one context to another. The purpose of grouping values as epistemic or non-epistemic is to distinguish those values that are, at a quite general level, beneficial from an epistemic point of view from those that are not. What counts as a (certain type of) value does not vary from one context to another. Values do not behave like that.

We of course agree with McMullin that some epistemic values in some contexts matter more than others. Scope is a much more important value in physics than in, for instance, structural engineering. The same goes for a value such as accuracy. In many contexts engineers prefer, and should prefer, the less accurate Newtonian mechanics instead of the more accurate relativistic mechanics. However, this point is perfectly consistent with our claim that the demarcation line between what counts as an epistemic and a non-epistemic value does not vary across different contexts. 
Now consider the following, revised definition:

Definition 2 An epistemic value is one which will, in each and every case, if pursued, help toward the attainment of at least one of the epistemic goals of science or engineering, whatever those goals are; all other values are nonepistemic values.

As far as we can see, this way of drawing the distinction makes the biconditional come out true. This is good news. However, a possible objection could be that we have just replaced one obscure term by another: what are the epistemic goals of science? In response to this objection, we would like to make two points. First, we note that although it might be hard to determine what the goals of science or engineering actually are, we all understand the meaning of that term quite well. Second, we note that a few general points about epistemic goals seem to be uncontroversial. For instance, the epistemic goals of a discipline are typically quite general. Although knowledge could be a candidate for an epistemic goal of science, it would be odd to claim that only knowledge about Newtonian mechanics or some other particular theory is an epistemic goal. Moreover, in engineering general "know how" about how to solve certain types of problems might qualify as an epistemic goal, but hardly "know how" about how to solve a particular design issue (although solving such issues can of course be important for a number of reasons). ${ }^{3}$

Given such restricted notion of epistemic goals, our definition helps us to clarify the distinction between epistemic and non-epistemic values, irrespective of whether we are able to determine once and for all exactly what the epistemic goals of science are. By adopting Definition 2 we attain the conceptual clarity we are looking for.

\section{The Role of Non-Epistemic Values in Modelling}

It is relatively easy to give examples of non-epistemic values that influence the construction of models in science and engineering. The list of non-epistemic values includes, for instance, safety, sustainability, equality, nonmaleficence, reliability, economic prosperity and wellbeing. However, before we discuss these values we would like to say a few words about the definition of a model. ${ }^{4}$

As we see it, models comprise at least propositions expressing scientific representations and propositions expressing empirical assumptions. The representational propositions express relations between all sorts of entities. For example, the model of the solar system includes representations of the planets and their relations to each other. The empirical assumptions ascribe properties to representations; for instance, many models of the solar system assume that planets are perfectly round. It is plausible to assume that every model is designed with a specific goal in mind. The goal of the model determines what kind of output the model should generate, e.g., a

\footnotetext{
${ }^{3}$ We wish to thank one of the reviewers for helping us to clarify this point.

4 See Diekmann (2011), "Moral Mid-Level Principles in Modeling” (manuscript), for a more detailed discussion of models.
} 
numerical value, or an evaluative judgment about a state, or a judgement about a characteristic behaviour.

Now, in order to bring home our point about the importance of non-epistemic values, we shall discuss four examples of models that have been constructed and used by engineers and scientists. These examples show that several quite different non-epistemic values influence the construction of models in science and engineering. 5

Example 1 Non-epistemic values influence the choice of represented properties.

Clausen and Cantwell (2007) describe the calculation of safety factors in decision-making tools. Safety factors are parameters, determined by rules of thumb that help to anticipate unforeseeable hazards. For illustrative purposes, Clausen and Cantwell discuss the calculation of the reference dose (RfD) used by toxicologists in risk assessments of chemicals. The non-epistemic, toxicological goal of the RfD is to identify a dose of a toxic substance that is sufficiently unlikely to put humans at risk. The RfD is calculated as follows:

$$
\operatorname{RfD}:=\frac{\text { NOAEL }}{U_{A} \cdot U_{H} \cdot U_{S} \cdot U_{D} \cdot M}
$$

where

(i) NOAEL, the "no observed adverse effect level", is the dose of the substance that has been shown not to be harmful to animals (e.g., rats);

(ii) $U_{A}$ is the interspecies factor, which reflects uncertainty due to physiological differences between humans and animals (a common value is 10);

(iii) $U_{H}$ is the intraspecies factor, which reflects uncertainty about differences among humans (a common value is 10);

(iv) $U_{S}$ is the chronicity factor, which is larger than 1 if the observations are shortterm while the RfD is used for long-term considerations (values are usually less than 10);

(v) $U_{D}$ is the database factor, which is included if a database is incomplete or insufficient (values vary between 1 and 100); and

(vi) $M$ is the modifying factor, which is used for covering unforeseen considerations (usually less than 10).

The formula for calculating the RfD highlights an important way in which nonepistemic values can influence a model. Safety, which is a paradigmatic example of a non-epistemic value, influences the choice of represented parameters. The NOAEL and all the uncertainties about substances listed above are included in the model, because they are deemed to be relevant for ensuring that the RfD guarantees safety in various contexts. If safety had not been a concern, this choice of the effect level (numerator) as well as the divisors would not have constituted an appropriate model.

Example 2 Non-epistemic values influence the thresholds used in models.

\footnotetext{
$\overline{5}$ We try to balance between models from science and models from engineering. Some examples are on the border between these two. Since a full-fledged distinction between engineering and science would not contribute to our point, we leave it out and emphasize our main point.
} 
The Knowledgeable Service Robots for Aging (KSERA) project (Salerno et al. 2010) researches home care systems for the elderly, in which fall detectors play an important role. Fall detectors are devices that are worn on the body and detect falls by measuring rapid changes in the movements.

The device detects a fall by recording four successive phases, namely (i) "weightlessness", (ii) "impact", (iii) "inactivity", and finally (iv) "difference to initial status". Each fall detector contains a small weight, for example $1 \mathrm{~g}$. In the first phase, weightlessness is detected if the weight is measured to weigh less than $1 \mathrm{~g}$. (This happens if the detector and weight fall together with the patient.) In the second phase, the impact phase, the weight accelerates heavily when the detector suddenly stops as the patient's body hits the ground. In the third phase, inactivity is recorded as the human body remains motionless on the ground for some time. Finally, the fourth phase is recorded if a change in orientation of the body (from standing to lying) is detected. If all four phases are recorded in this particular sequence, then the detector sets off an alarm, alerting a healthcare provider that a patient has fallen in his or her home and needs assistance.

The fall model used in the construction of the detector comprises at least one non-epistemic value-judgment. In order to see this, note that the detector has to be programmed to set off the alarm, only if the recorded data exceed certain thresholds. What the appropriate level of these thresholds is, is by no means a purely epistemic question. It is very reasonable to also take the patient's wellbeing into account when setting the thresholds. For instance, a very sensitive detector (i.e., a device that produces a large number of false positives) would cause a lot of irritation for the patient and perhaps even remain unused, which would affect the patient's wellbeing negatively in the long run. For analogous reasons, a device that is not sufficiently sensitive (i.e., a device that produces a large number of false negatives) could fail to detect a fall and could thus affect the patient's wellbeing negatively. In some situations, considerations of wellbeing of course have to be balanced against other, purely epistemic considerations. Thus, wellbeing is a non-epistemic value that sometimes influences the construction of models, such as fall models.

Example 3 Non-epistemic values counterbalance the influence of epistemic values.

Jenkins and McCauley (2006) discuss Geographical Information Systems (GIS) used for supporting decision-makers in geo-engineering. The goal of a GIS is to model the topography of a landscape, including water streams and height differences of the ground. The landscape is represented as a data grid of cells with different heights on an integer scale. Each cell is categorized as terrestrial (e.g., forests) or aquatic (rivers and lakes). In nearly all GIS models it is assumed that, (i) water streams are fractal systems, and that (ii) single cells in the data grid that are lower than the surrounding ones are data noise. (p. 278)

The assumption that water streams have fractal geometry, i.e., assumption (i), is powerful but restrictive, because fractal geometries comprise repeated patterns on different scales. Trees typically have a fractal geometry: if one cuts a big branch from a naturally grown tree and compares it to the whole tree, then these two pieces have pretty much the same structure. Moreover, if one breaks off one of the sub-branches from the big branch, one will again discover that the tree, the big 
branch, and the sub-branch have roughly the same structure. The same holds true for many natural objects such as plants, coasts, or rivers. By assuming fractal geometry only a small number of data points are required for generating accurate representations of artefacts exhibiting this pattern.

Assumption (ii), according to which single data cells that are lower than the surrounding ones should be treated as noisy data, is included in GIS models because most single data cells are due to rounding or sampling errors (p. 278). Rounding errors occur because a GIS represents heights in integers, in contrast to the floating points that are used for collecting the measurements. Sampling errors occur in measurements of the actual terrain, if the height of the measured point does not represent the surrounding height. For example, a hollow in the ground does not represent the actual height of its surrounding.

Jenkins and McCauley correctly point out that assumptions (i) and (ii) are problematic because they entail that wetlands are not accurately represented in GIS models. Wetlands are parts of the water flow system with central ecological functions, and '[t]hese wetlands ... provide important ecological services such as flood mitigation, groundwater recharge, nutrient processing, and habitat for unique flora and fauna.' (p. 278) Since wetlands do not fulfil the requirements of fractal geometry, and since many wetlands are smaller than a single grid cell, assumptions (i) and (ii) entail that wetlands are not accurately represented in GIS models.

This neglect of wetlands causes a value conflict. Briefly put, assumptions (i) and (ii) are made with the intention to increase the predictive power and accuracy of the model; both are epistemic values. Therefore, from a purely epistemic point of view assumptions (i) and (ii) may very well be warranted. However, since it is widely accepted that a non-epistemic value such as sustainability is relevant when employing GIS models for landscape management (which requires that wetlands are accurately represented), it becomes clear that the representation's predictive power and accuracy conflict with sustainability.

Example 4 Non-epistemic values influence the choice of models.

Fienberg (1994) discusses the choice between statistical models used in the US decennial census. The US population is counted every 10 years and the results are used to allocate, among other things, Congressional seats and federal funds. A major problem in the decennial census is how to deal with ethnic minorities, which are less likely to be counted than others. This gives rise to a serious undercount problem. The problem is deeply rooted in social and socioeconomic problems, but needs to be tackled with statistical methods. An unresolved undercount problem will typically lead to economic benefits for a socially strong group of white middle class Americans.

In the decennial census in 1990 two rival statistical models were considered. The Bureau of Census proposed a model that they argued would lead to statistical improvements at the global data level. A group of independent statisticians proposed alternative models designed to reduce the effect of the undercount problem at the regional level. (p. 131) While the Bureau's method was never questioned, the statistical models developed by the independent statisticians went through a radical development process in the period leading up to the 1990 census and eventually became widely accepted among statisticians. 
A few years before the 1990 census, a decision was made not to use the model developed by the independent statisticians. According to Fienberg, the actual reason for not using the improved statistical methods had nothing to do with epistemic considerations, but was entirely motivated by political and economic considerations, such as profit. Fienberg furthermore argues that for reasons of equality the independently developed model should had been employed instead. Let us suppose that these claims are correct. Then, the upshot of this example is that sometimes, such as in this particular census, the choice of model is directly affected by nonepistemic, moral and political values.

\section{Should Non-Epistemic Values be Excluded?}

It is hardly surprising that non-epistemic values actually play a role in many models used in science and engineering. As shown above, the problem that a model seeks to address can seldom be resolved by taking only epistemic considerations into account. However, it does not follow from this that non-epistemic values ought to influence models. Many scientists and engineers believe that models are epistemic tools for scientific inquiry, and that models and their output should therefore not be influenced by non-epistemic values. We, however, reject this claim.

We believe, partly in line with what Rudner (1953) sketched in his classic article, that when models are used for solving non-epistemic problems, then non-epistemic values partly determine whether a model ought to be accepted or rejected. Our argument is based on the premise that models are, and ought to be, developed with a certain goal in mind. Sometimes the goal is purely epistemic, such as in certain branches of physics and chemistry. However, there are also cases in which the goal of the model is, at least partly, non-epistemic. For example, the fall detector discussed in Example 2 seeks to increase the safety and wellbeing of elderly. The detector uses a model of what should "count" as a fall. However, as explained in Example 2, a purely epistemic approach to what should count as a fall is not what engineers look for, or should be looking for, in this case. A number of non-epistemic value-judgements about safety and wellbeing also matter. When constructing the fall model, engineers should therefore not just focus on the epistemic description of the fall. This means that what counts as a successful model partly depends on the fulfilment of non-epistemic goals.

Our general argument can be summarised as follows:

(i) Models are, and ought to be, developed with one or several goals in mind.

(ii) Sometimes one of the goals is, and ought to be, a non-epistemic goal.

(iii) The extent to which a non-epistemic goal is accurately reflected in a model depends on the influence of non-epistemic values.

(iv) Some models are influenced, and ought to be influenced, by non-epistemic values. ${ }^{6}$

\footnotetext{
${ }^{6}$ Note that we are by no means trying to derive an 'ought' from an 'is' here. According to our argument, it is not the mere fact that models are developed with a certain goal in mind that justifies the conclusion that models sometimes ought to reflect non-epistemic values. The 'ought' in the conclusion of the
} 
While we have already discussed the first two premises in the paragraph above, the core of the argument lies in the third premise. In order to understand the role that value influences play in the third premise, it is helpful to note that the relation between the model and the modelled entity is asymmetric. Every model represents or describes another entity, whereas the entity does not represent or describe the model (cf. Suárez 2003, p. 232). This asymmetry is what lies behind the popular slogan 'all models are wrong'. In essence, there are always some aspects of the entity that are not covered by the model.

When a modeller seeks to achieve his or her goals, the asymmetry described above entails that it will always be possible to construct rival models, which seek to solve the same problem in different ways. However, if one of the rival models does not contribute to solving the goal, there is no reason to adopt it. Moreover, how well each rival model performs depends on what is considered to be desirable or undesirable in light of the goals of the model. Therefore, when there are choices to be made between rival models, these choices will depend on value-judgments. ${ }^{7}$

In addition to selecting a model from a set of rival models, at least two further types of value-judgments ought to affect the construction of models, viz. valuejudgments that determine choices about which properties of the modelled entity are to be represented, and value-judgments that determine which assumptions are to be made. The choice of the represented properties influences whether all issues are respected that are relevant to the goal. For example, how safe the RfD actually is hinges on the choice of the particular safety factors. The assumptions in a model can ease the achievement of the goal, but they can also contradict or inflict with it. In the GIS example, the assumptions inflict with the goal of promoting sustainability. Both value-judgments are thus essential for attaining the goal of the model.

Turning away from value-judgments back to values, we concede that for purely epistemic goals, scientists and engineers need not, and typically do not, take nonepistemic values into account. However, in many cases at least one of the goals is non-epistemic. For instance, the goal behind constructing models for fall detectors is to increase wellbeing of elderly people (see Example 2). Whether the fall model is a perfectly accurate representation of falls is thus not its major concern. As said above, a good representation of falls is a mere means to the actual end of increased wellbeing. Therefore, engineers who choose a model for constructing a fall detector have to trade-off epistemic goals, such as fall-representation, against non-epistemic goals, such as increased wellbeing. The value-judgments that are made when developing and accepting the fall model will allow for such trade-off towards increased wellbeing, only if also non-epistemic values, such as wellbeing, determine these judgments. The same holds true for value-judgments in general. Only if non-epistemic values influence what is desired, the non-epistemic aspects of

Footnote 6 continued

argument comes from the 'ought' in the premise, i.e., the claim that models ought to be developed partly with non-epistemic goals in mind, including goals such as safety and wellbeing.

7 As a commentator pointed out, it is possible to see models as social constructs. Under this particular condition, the role of non-epistemic values becomes even more apparent: The involved persons are usually not just scientists or engineers, but also users. Therefore the desirabilty of a model always depends also on user-related non-epistemic considerations and connected non-epistemic values. 
non-epistemic goals can be fully satisfied in a value-judgment. Hence, to which extent the non-epistemic goals of the model are promoted by value-judgments depends on non-epistemic values.

For this reason, non-epistemic values influence and ought to influence the construction and choice of models. Given a non-epistemic goal, non-epistemic values are needed for pursuing that goal. Since value-judgments are always involved in the construction of a model, and value-judgments are needed for deciding which model it is best to employ, non-epistemic values should determine which model is chosen. $^{8}$

\section{Concluding Discussion}

Many engineers and scientists believe that models should be entirely value-free, or that models should at least contain no non-epistemic values. However, this traditional approach fails to recognize that in the applied sciences, and in many branches of engineering, the goals that we seek to achieve are not purely epistemic. We have argued that in order to relate models to non-epistemic goals, models should be partly determined by non-epistemic values. Moreover, non-epistemic values are not just "secondary values" that become important once all the epistemic values have been taken into account. Non-epistemic values can actually be in direct conflict with epistemic values, as seen in the GIS case (Example 3). Thus, for non-epistemic reasons, non-epistemic value-judgments should influence the construction of models and the choice between rival models should partly depend on non-epistemic values.

We believe that the implications for modelling practice can be far-reaching. For instance, we would like to encourage scientists and engineers to change their perspective and focus more on a context sensitive approach. When developing a fall model, for instance, the engineer should focus less on modelling the fall itself, but rather try to find a model that optimizes wellbeing. Moreover, in future research on the role on non-epistemic values in modelling it might be reasonable to apply insights from the value-sensitive design approach discussed by e.g., van den Hoven and Manders-Huits (2009); see also van de Poel (2009).

Finally, let us just mention that many rules of good practice or professional norms are already implicitly based on non-epistemic values and conventions.

\footnotetext{
${ }^{8}$ As an alternative view, one could state that all non-epistemic aspects can be formulated as epistemically assessable terms. Such formulation would be prior to the actual model construction. Then, epistemic values would be sufficient to develope and evaluate the performance of models. See e.g., the RfD, Example 1: One would first define which doses are regarded as safe, which uncertainties to include, and how big the safety factors should be chosen. Consequently, tracing an RfD would remain a solely epistemic issue (namely measuring the NOAEL and calculating the RfD). Hence, so the reasoning goes, one could seek to exclude non-epistemic values and argue that models ought to be free of non-epistemic values.

However, such an alternative view distorts the concept of models. The strategy of the alternative view is to simply redefine the notion of a model: All non-epistemic influences on models are excluded as parts that are prior to models. The problem is, however, that this strategy neglects that definitions and assumptions are a fundamental part of models. Without defining which entities are described or represented and without assuming simplified properties of those entities, models could neither describe nor represent. Indeed, they would not distinguish from formulae, algorithms, or calculations.
} 
Questioning and overcoming those conventions could help scientists and engineers to be case-sensitive and to optimally address all involved non-epistemic issues.

Acknowledgments We would like to thank our colleagues in the Section for Philosophy and Ethics at Eindhoven University of Technology, the members of EET at RWTH Aachen, and the members of the OZSE working group for ethics of technology for helpful and inspiring comments on earlier drafts.

Open Access This article is distributed under the terms of the Creative Commons Attribution Noncommercial License which permits any noncommercial use, distribution, and reproduction in any medium, provided the original author(s) and source are credited.

\section{References}

Clausen, J., \& Cantwell, J. (2007). Reasoning with safety factor rules. Techné: Research in Philosophy and Technology, 11(1), 55-70.

Dorato, M. (2004). Epistemic and nonepistemic values in science. In P. Machamer \& G. Wolters (Eds.), Science values and objectivity (pp. 52-77). Pittsburgh: University of Pittsburgh Press.

Fienberg, S. E. (1994). Ethical and modeling considerations in correcting the results of the 1990 decennial census. In W. A. Wallace (Ed.), Ethics in modeling (pp. 103-144). Oxford, UK: Elsevier Science Ltd.

Jenkins, D. G., \& McCauley, L. A. (2006). GIS, SINKS, FILL, and disappearing wetlands: Unintended consequences in algorith development and use. In Proceedings of the 2006 ACM symposium on applied computing (pp. 277-282). ACM.

Kuhn, T. S. (1962). The structure of scientific revolutions. Chicago: Chicago University Press.

Kuhn, T. S. (1977). Objectivity, value judgment and theory choice. The essential tension: Selected studies in the scientific tradition and change (pp. 356-367). Chicago: University of Chicago Press.

McMullin, E. (1982). Values in science. In P. K. Machamer \& G. Wolters (Eds.), PSA: Proceedings of the biennial meeting of the philosophy of science association, Vol. 2: Symposia and invited papers (pp. 3-28). Chicago: The University of Chicago Press.

Rudner, R. (1953). The scientist qua scientist makes value judgments. Philosophy of Science, 20(1), 1-6.

Salerno, C., Feletti, L. C., \& Indino, D. (2010). Learning \& decision making algorithms in pervasive environments. Public document of the KSERA project. http://cms.ieis.tue.n1/ksera/documents/ KSERA_D4.1.pdf. Accessed April 8, 2011.

Steel, D. (2010). Epistemic values and the argument from inductive risk. Philosophy of Science, 77(1), 14-34.

Suárez, M. (2003). Scientific representation: Against similarity and isomorphism. International Studies in the Philosophy of Science, Routledge, 17(3), 225-244.

van de Poel, I. (2009). Values in engineering design. In A. Meijers (Ed.), Handbook of the philosophy of science. Volume 9: Philosophy of technology and engineering sciences (pp. 973-1006). Amsterdam: North Holland.

van den Hoven, J., \& Manders-Huits, N. (2009). Value-sensitive design. In J. K. B. Olsen, S. A. Pedersen \& V. F. Hendricks (Eds.), A companion to the philosophy of technology (pp. 477-480). West Sussex: Wiley.

Walker, W. E. (2009). Does the best practice of rational-style model-based policy analysis already include ethical considerations? Omega, 37, 1051-1062. 